\title{
Brain hypermetabolism in amyotrophic lateral sclerosis: a FDG PET study in ALS of spinal and bulbar onset
}

\author{
Angelina Cistaro • Maria Consuelo Valentini • Adriano Chiò • Flavio Nobili • \\ Andrea Calvo • Cristina Moglia • Anna Montuschi • Silvia Morbelli • Dario Salmaso • \\ Piercarlo Fania • Giovanna Carrara • Marco Pagani
}

Received: 26 July 2011 / Accepted: 14 October 2011 /Published online: 17 November 2011

(C) Springer-Verlag 2011

\begin{abstract}
Purpose To identify the neurobiological traits of amyotrophic lateral sclerosis (ALS) and to elucidate functional differences between ALS of spinal and bulbar onset. We hypothesized that glucose metabolism distribution might vary between groups.

Methods The study groups comprised 32 patients with ALS of either bulbar $(n=13)$ or spinal $(n=19)$ onset and 22 subjects as controls. They were investigated by $\left[{ }^{18} \mathrm{~F}\right]$ fluorodeoxyglucose (FDG) positron emission tomography (FDG PET), comparing the patient groups with each other and with the controls by statistical parametric mapping.

Results Highly significant relative increases in glucose metabolism distribution were found in the group comprising all 32 ALS patients as compared with the controls in the bilateral amygdalae, midbrain, pons and cerebellum. Relative hypermetabolism was also found in patients with spinal onset as compared with the controls in the right midbrain. In patients with bulbar onset
\end{abstract}

\footnotetext{
A. Cistaro $\cdot$ P. Fania

Positron Emission Tomography Center IRMET S.p.A,

Turin, Italy

M. C. Valentini · G. Carrara

Department of Neuroradiology, CTO Hospital,

Turin, Italy

A. Chiò $\cdot$ A. Calvo $\cdot$ C. Moglia $\cdot$ A. Montuschi

Department of Neuroscience, ALS Center, University of Turin,

Turin, Italy

F. Nobili

Department of Neurosciences, Clinical Neurophysiology Unit, Ophthalmology and Genetics, University of Genoa,

Genoa, Italy
}

compared with the controls and with patients with spinal onset, large relatively hypometabolic areas were found in the bilateral frontal cortex, right insula, anterior cingulate, precuneus and inferior parietal lobe. Patients with spinal onset had significantly higher scores in a neuropsychological test assessing verbal fluency compared with patients with bulbar onset.

Conclusion This large FDG PET investigation provided unprecedented evidence of relatively increased metabolism in the amygdalae, midbrain and pons in ALS patients as compared with control subjects, possibly due to local activation of astrocytes and microglia. Highly significant relative decreases in metabolism were found in large frontal and parietal regions in the bulbar onset patients as compared with the spinal onset patients and the controls, suggesting a differential metabolic and neuropsychological state between the two conditions.

Keywords Positron emission tomography - Amyotrophic lateral sclerosis $\cdot$ Bulbar onset $\cdot$ Spinal onset

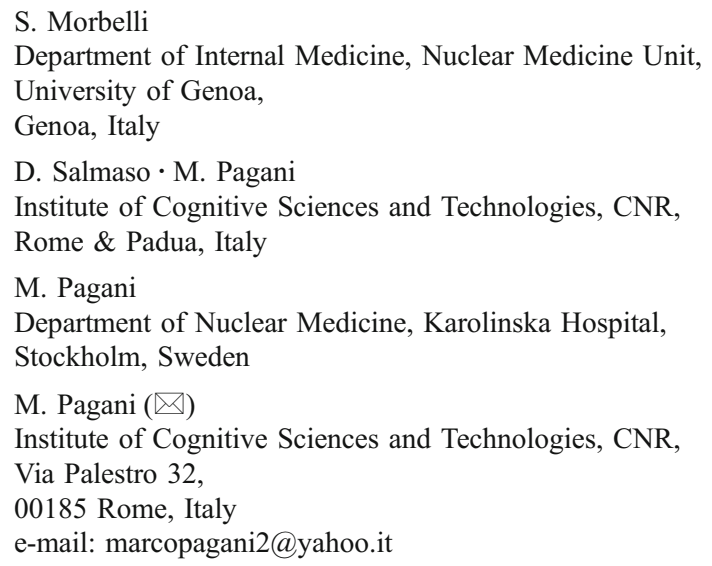




\section{Introduction}

Amyotrophic lateral sclerosis (ALS) is a fatal late-onset neurodegenerative disorder of adult life, characterized by a progressive impairment of motor function at the bulbar and spinal levels. Described for the first time by Charcot in 1869, it is characterized by selective loss of cells in the anterior horns of the spinal cord and in the lower motor cranial nerve nuclei as well as by corticospinal tract degeneration. The clinical diagnosis of ALS is still based on clinical history and neurological examination and is supported by neuropsychological examination and by the exclusion of other disorders [1]. It is usually delayed by 1 year after a patient's first referral. The incidence of ALS is 2-3/100,000 new cases per year [2]. The onset is at the spinal level (with weakness and hypotrophy of the muscles of upper or lower limbs) in two-thirds of patients and at the bulbar level (with dysphagia and dysarthria) in onethird of patients [2]. However, increasing evidence suggests that ALS also affects extramotor systems and may be regarded as a multisystem disorder [3-5]. Executive [4, 6, 7] and memory $[8,9]$ deficits have been detected using a variety of neuropsychological tests. Furthermore, impaired verbal fluency has been correlated with the attenuated response during a motor paradigm in several frontal and subcortical regions independently of physical disability [9-11]. A reduction in extramotor white matter volume has been found in cognitively impaired ALS patients, especially in regions corresponding to the frontal and frontotemporal association fibres [11, 12] in which white matter hyperintensities have also been found with magnetic resonance imaging $[13,14]$.

In some early $\left[{ }^{18} \mathrm{~F}\right] 2$-fluoro-2-deoxy-D-glucose (FDG) positron emission tomography (PET) studies, ALS was associated with frontal lobe dysfunction [15] and eventually dementia [16]. A pioneering study in a limited number of patients showed a generalized metabolic reduction in cortex and basal ganglia which worsened with progression of the disease in ALS patients with predominant upper motor neuron signs but not in patients with predominant lower motor neuron signs [17]. A further finer analysis in the same cohort of patients highlighted a larger degree of hypometabolism in the motor sensory area as well in the parietal and occipital cortex [18]. However, Ludolph et al. [7] found a diffuse reduction in frontal and occipital cortical FDG uptake in 18 ALS patients displaying upper and lower motor neuron signs and mild executive dysfunction without a significant correlation between glucose consumption and disease and clinical neurological deficit duration. Moreover, reduced FDG and $\left[{ }^{11} \mathrm{C}\right]$-flumazenil binding have been seen in rolandic and perirolandic areas [19-21] in three different PET studies investigating primary lateral sclerosis.

Taken together these findings suggest that extramotor structures of the central nervous system are affected in ALS. In particular, there is growing evidence suggesting that frontal lobes are impaired in some patients. However, currently no neuroimaging technique provides positive support for the diagnosis of ALS.

The aim of this study was to use PET to investigate FDG brain distribution in a large cohort of ALS patients characterised by bulbar or spinal onset. Based on previous literature suggesting differences in extramotor white and grey matter involvement in bulbar and spinal ALS, we hypothesized that glucose metabolism distribution might vary between the two groups of patients and differentiate the two forms of ALS neurobiologically.

\section{Materials and methods}

\section{Participants}

The ALS patients comprised 15 women and 17 men (mean age 63.3, SD 13.1, range 85-42 years; disease duration $17.8 \pm 16$ months) seen consecutively at the Turin ALS Centre where they had been referred for FDG PET. The patients received probable laboratory-supported or definite ALS diagnosis according to the revised El Escorial ALS criteria [1]. Patients were classified according to the site of onset: bulbar $(n=13)$ or spinal $(n=19)$. The presence of frontotemporal cognitive and behavioural syndromes in ALS was assessed with a battery of neuropsychological tests with an emphasis on executive functions including the phonological verbal fluency test (FAS), the trail making test (TMT, forms A and B), the Stroop colour-word test and the Wisconsin card-sorting test [22], which were scored according to Italian normative values, correcting for age and level of education. Depression was evaluated using the hospital anxiety and depression scale.

The control group comprised 8 women and 14 men (mean age 62, SD 14, range 80-34 years) found to be completely free of disease by ${ }^{18}$ F-FDG PET and with a normal neurological assessment. Exclusion criteria were the presence of major systemic illness, major vision disturbances, psychiatric illnesses, epilepsy, head trauma, parkinsonism, previous stroke or TIA, and a brain mass as well as current use of benzodiazepines and tricyclic antidepressants.

All participants signed a written informed consent form in accordance with the principles outlined in the Declaration of Helsinki.

\section{${ }^{18}$ F-FDG PET}

Subjects fasted for at least 6 hours. Before radiopharmaceutical injection, blood glucose was measured ( $<130 \mathrm{mg} / \mathrm{dl}$ in all patients). After a 10-min rest in a silent and darkened room, with eyes closed and ears 
Table 1 Executive function tests: scores and significance of differences. Values are mean scores \pm SD

\begin{tabular}{llll}
\hline Test & Spinal group $(n=19)$ & Bulbar group $(n=13)$ & $p$ value \\
\hline FAS & $37.9 \pm 12.8$ & $24.8 \pm 11.8$ & 0.006 \\
TMT-A & $50.3 \pm 39.0$ & $64.10 \pm 42.6$ & NS \\
TMT-B & $64.1000 \pm 66.9$ & $126.8889 \pm 90.4$ & 0.046 \\
TMT B-A & $18.4500 \pm 42.8$ & $66.8889 \pm 71.4$ & 0.031 \\
Stroop word & $11.9000 \pm 3.6$ & $5.8000 \pm 3.5$ & 0.000 \\
Stroop colour & $7.9000 \pm 3.3$ & $7.2000 \pm 4.0$ & $\mathrm{NS}$ \\
Stroop colour-word & $6.4000 \pm 3.4$ & $6.5556 \pm 4.1$ & $\mathrm{NS}$ \\
Wisconsin card sorting & $63.9421 \pm 40.5$ & $85.0000 \pm 28.4$ & $\mathrm{NS}$ \\
\hline
\end{tabular}

unplugged, the subjects were injected with approximately $185 \mathrm{MBq}$ of ${ }^{18} \mathrm{~F}-\mathrm{FDG}$ through an intravenous cannula. PET/CT scanning was started approximately 60 min after the injection and lasted for a further $10 \mathrm{~min}$. A polycarbonate head holder was applied to reduce head movements during the scan.

The PET/CT scans of the brain were acquired using a Discovery STE system (GE Healthcare). FDG PET/CT images were acquired with two sequential scans: a CT scan (thickness $3.75 \mathrm{~mm}, 140 \mathrm{kV}, 60-80 \mathrm{mAs}$ ) and a PET scan (one transaxial FOV of $30 \mathrm{~cm}$ ). The PET scan was initiated immediately after the CT scan, in order to use the CT data for attenuation correction of the PET data. Data were collected in $128 \times 128$ matrices with a reconstructed voxel of $2.34 \times 2.34 \times 2.00 \mathrm{~mm}$.

\section{Statistical analysis}

A two-sample unpaired $t$-test was used to differentiate groups based on age and executive tests scores, while the chisquared test was used to evaluate gender differences. Using statistical parametric mapping (SPM2; Wellcome Department of Cognitive Neurology, London, UK) implemented in Matlab 6.5 (Mathworks, Natick, MA), PET data were subject to affine and nonlinear spatial normalization into the MNI space. The spatially normalized sets of images were then smoothed with an 8-mm isotropic gaussian filter to blur individual variations in gyral anatomy and to increase the signal-to-noise ratio. The resulting statistical parametric maps, $\operatorname{SPM}\{\mathrm{t}\}$, were transformed into normal distribution $(\operatorname{SPM}\{z\})$ units. SPM coordinates were corrected to match the Talairach coordinates by the subroutine implemented by Matthew Brett (http://brainmap.org/index.html). Brodmann areas (BAs) were then identified at a range of 0 to $3 \mathrm{~mm}$ from the corrected Talairach coordinates of the SPM output isocentres, after importing the corrected coordinates, by Talairach client (http://www.talairach.org/index.html).

Following Bennett et al. [23], the $p<0.05$ threshold, corrected for multiple comparisons with the False Discovery Rate (FDR) option, was used to explore SPM $t$-maps at the voxel level. If statistical significance was not reached, the threshold at the voxel level was explored at $p<0.001$ uncorrected for multiple comparisons. The significance of the identified area was established at $p<$

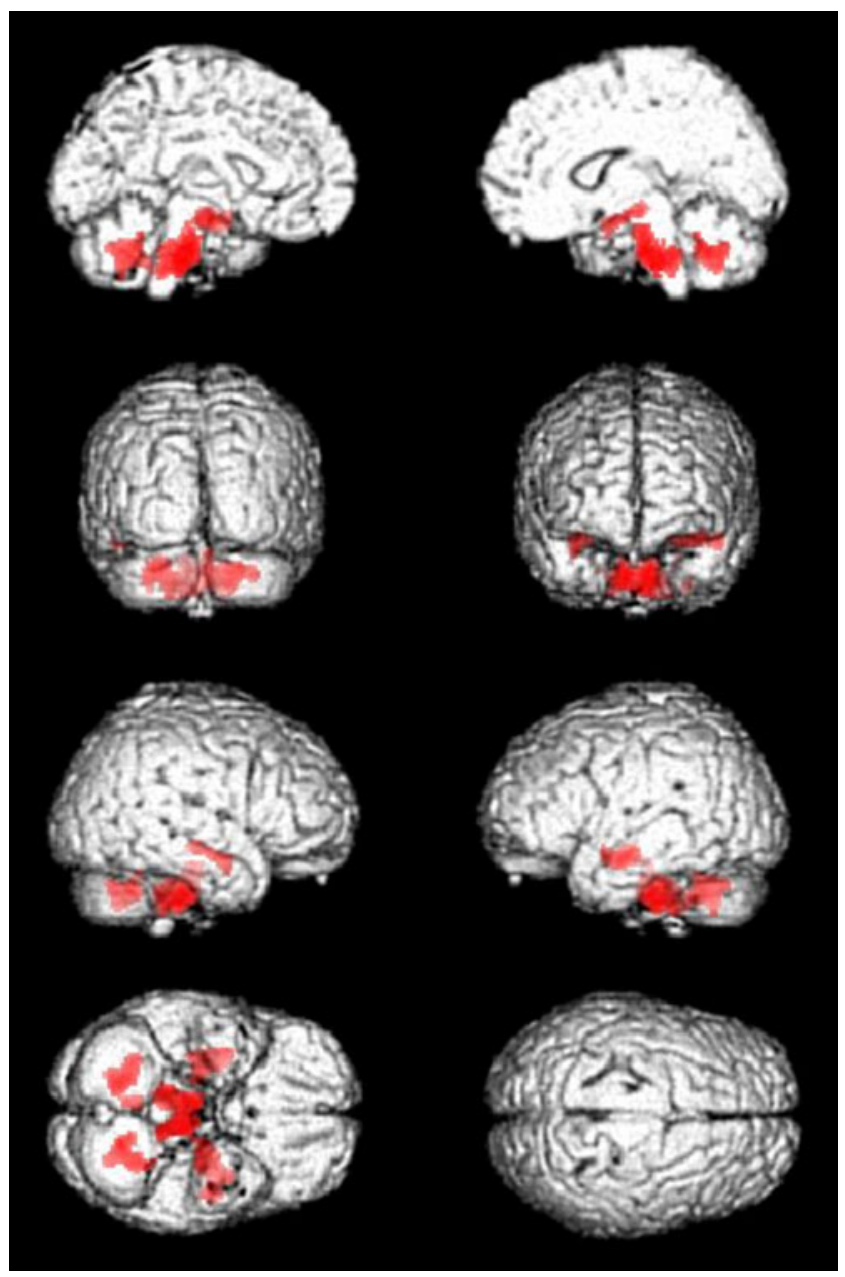

Fig. 1 Three-dimensional rendering showing those regions in which ${ }^{18}$ F-FDG uptake was significantly higher in ALS patients $(n=32)$ than in the controls $(n=22)$ (threshold $p<0.05$, corrected for multiple comparisons with the FDR at the voxel level): top row left medial left view; top row right medial right view; second row left posterior view; second row right frontal view; third row left right-side view; third row right left-side view; bottom row left view from below; bottom row right view from above. Coordinate and regional details are presented in Table 2 
Table 2 Numerical results of SPM comparisons between ${ }^{18}$ F-FDG uptake in the controls and ALS patients

\begin{tabular}{|c|c|c|c|c|c|c|c|}
\hline \multirow[t]{2}{*}{ Comparison } & \multicolumn{3}{|l|}{ Cluster level } & \multicolumn{4}{|l|}{ Voxel level } \\
\hline & $\begin{array}{l}\text { Cluster extent } \\
\text { (no. of voxels) }\end{array}$ & $\begin{array}{l}\text { Corrected } \\
p \text { value }^{\mathrm{a}}\end{array}$ & $\begin{array}{l}\text { Cortical region } \\
\text { of voxel }\end{array}$ & Maximum $Z$ score & $\begin{array}{l}\text { Talairach } \\
\text { coordinates }\end{array}$ & Cortical region $^{\mathrm{b}}$ & $\mathrm{BA}^{\mathrm{b}}$ \\
\hline \multirow{8}{*}{$\begin{array}{l}\text { Controls } \\
\text { vs. ALS patients }\end{array}$} & \multirow[t]{4}{*}{256} & \multirow[t]{4}{*}{0.009} & Left frontal & 4.11 & $-40,7,35$ & Precentral gyrus & 9 \\
\hline & & & Left frontal & 4.00 & $-33,8,55$ & Middle frontal gyrus & 6 \\
\hline & & & Left frontal & 3.80 & $-42,10,42$ & Middle frontal gyrus & 8 \\
\hline & & & Left occipital & 3.67 & $-5,-74,-5$ & Lingual gyrus & 18 \\
\hline & \multirow[t]{4}{*}{433} & \multirow[t]{4}{*}{0.001} & Right frontal & 4.56 & $32,-17,59$ & Precentral gyrus & 6 \\
\hline & & & Right occipital & 3.74 & $23,-79,-12$ & Fusiform gyrus & 19 \\
\hline & & & Right occipital & 3.45 & $21,-89,12$ & Middle occipital gyrus & 18 \\
\hline & & & Right occipital & 3.41 & $23,-84,13$ & Cuneus & 17 \\
\hline \multirow{11}{*}{$\begin{array}{l}\text { ALS patients } \\
\text { vs. controls }\end{array}$} & \multirow[t]{6}{*}{1291} & \multirow[t]{6}{*}{0.000} & Left sublobar & 4.85 & $-23,-12,-10$ & \multirow[t]{2}{*}{ Amygdala } & \\
\hline & & & Left pons & 4.58 & $-6,-19,-30$ & & \\
\hline & & & Left temporal & 3.83 & $-43,-9,-14$ & \multirow[t]{2}{*}{$\begin{array}{l}\text { Superior temporal } \\
\text { gyrus }\end{array}$} & \multirow[t]{4}{*}{21} \\
\hline & & & Left midbrain & 3.42 & $-8,-22,-18$ & & \\
\hline & & & Left posterior & 3.38 & $-25,-62,-31$ & Pyramis & \\
\hline & & & Left posterior & 3.27 & $-25,-39,-41$ & Cerebellar tonsil & \\
\hline & \multirow[t]{5}{*}{379} & \multirow[t]{5}{*}{0.002} & Right midbrain & 4.69 & $7,-18,-19$ & & \\
\hline & & & Right pons & 4.52 & $7,-21,-30$ & & \\
\hline & & & Right posterior & 3.53 & $5,-51,-31$ & Cerebellar tonsil & \\
\hline & & & Right limbic & 3.36 & $31,-4,-12$ & Amygdala & \\
\hline & & & Right sublobar & 3.27 & $21,-18,-6$ & Lateral globus pallidus & \\
\hline
\end{tabular}

${ }^{a}$ Threshold $p<0.05$ corrected for multiple comparisons with the FDR at the voxel level. A value of $\leq 0.05$, corrected for multiple comparison at the cluster level, was accepted as statistically significant.

${ }^{\mathrm{b}}$ The corresponding cortical region and BA are reported for each significant cluster. If the maximum correlation is achieved outside the grey matter, the nearest grey matter (within a range of $3 \mathrm{~mm}$ ) is indicated with the corresponding BA

0.05 corrected for multiple comparisons at the cluster level. Only clusters containing more than 64 voxels were considered to be significant. The following comparisons were evaluated: (1) control vs. ALS and vice versa; (2) control vs. bulbar and vice versa; (3) control vs. spinal and vice versa; and (4) bulbar vs. spinal and vice versa. All the analyses were performed using age and gender as 'nuisance' variables.

Following the finding of relative hypermetabolism in all patient groups in comparison with the controls, all analyses were repeated in a post-hoc analysis by modifying the 0.8 default SPM value of the 'grey matter threshold'. This value is a grey matter uptake cut-off value that is included as a reference value in the global mean normalization step of SPM. With the threshold set to 0.8 , voxels with a mean value less than $80 \%$ of the mean are excluded. We thus increased this number to 1.0 in order to exclude white matter voxels and voxels from hypometabolic regions to minimize the risk that findings indicating hypermetabolism were an artefact of global mean normalization [24].

\section{Results}

No significant differences were found in age and sex distribution between patients and controls or between the bulbar and spinal groups. Of all the neuropsychological tests exploring executive functions, the FAS, the TMT-B, the TMT B-A and the Stroop word tests showed statistically significant difference between the spinal and bulbar groups, with the latter group scoring worse (Table 1).

Controls vs. all patients

Large clusters of relative hypermetabolism in the ALS patients as compared with the controls were identified, including the bilateral amygdalae, midbrain, pons and cerebellar tonsils, as well as the right lateral globus pallidus (Fig. 1, Table 2). The reverse comparison showed relatively hypometabolic clusters in ALS patients in the bilateral premotor cortex (BA 6) and lingual gyrus (BA 18), in the right primary visual cortex (BA 17) and fusiform gyrus (BA 19), and in the left precentral gyrus (BAs 8 and 9; Table 2). 


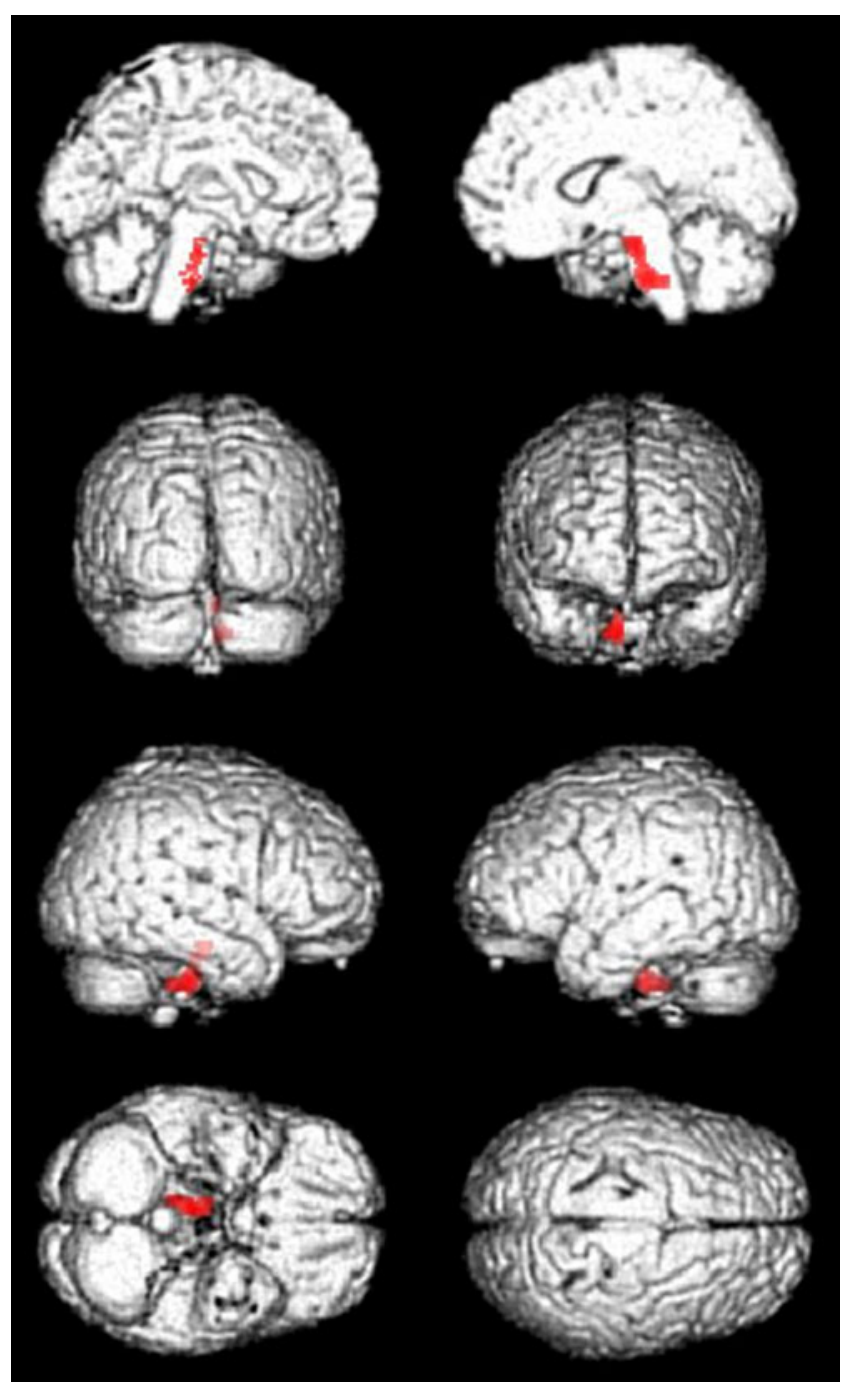

Fig. 2 Three-dimensional rendering showing those regions in which ${ }^{18}$ F-FDG uptake was significantly higher in SPI $(n=19)$ as compared with CTR $(n=22)$ (threshold $p<0.001$, uncorrected for multiple comparisons at voxel level): top row left medial left view; top row right medial right view; second row left posterior view; second row right frontal view; third row left right-side view; third row right leftside view; bottom row left view from below; bottom row right view from above. Coordinate and regional details are presented in Table 3

\section{Controls vs. spinal group}

Statistically significant relative hypermetabolism was found bilaterally in the spinal group in the right midbrain and pons as compared with the controls (Fig. 2; Table 3). Relative hypometabolism was found in the spinal group in small clusters in the bilateral lingual gyrus (BA 18) and in the right fusiform gyrus (BA 19) (Table 3).

Controls vs. bulbar group

Relative hypermetabolism was found in the bulbar group in a small cluster of voxels limited to the bilateral pons
(Table 4). On the other hand, a large cluster of voxels was found to be relatively hypometabolic in the bulbar group as compared to the controls in the bilateral prefrontal cortex (BAs 9, 46 and 47) and the premotor cortex (BA 6) as well as in the right insula (BA 13), anterior cingulate (BA 24), precuneus (BA 39) and inferior parietal lobe (BA 40) (Fig. 3, Table 4).

\section{Bulbar vs. spinal group}

Large relatively hypometabolic clusters were found in bulbar patients as compared with spinal patients in large prefrontal and frontal regions superimposed on those found to be relatively hypometabolic in the control vs. bulbar comparison. No significant differences were found when spinal group data were subtracted from bulbar group data.

All findings were confirmed by post-hoc analysis, which showed minimal changes in the number of significant voxels or in the $Z$-scores when the FDR option was used.

\section{Discussion}

The present study is to the best of our knowledge the largest ${ }^{18} \mathrm{~F}$-FDG PET investigation in ALS, taking into account the functional differences between patients with bulbar and spinal onset. The results strongly suggest a differential metabolic state between the two conditions, the common finding being the relative increase in metabolism in subcortical structures as compared to control subjects, described here for the first time. The second important finding is the highly significant relative decrease in metabolism in the large prefrontal, frontal and parietal regions in the bulbar onset group as compared with the spinal onset and control groups. The rather unexpected relative increase in ${ }^{18} \mathrm{~F}-\mathrm{FDG}$ uptake in our study was specifically located in the bilateral midbrain and pons, two regions containing corticospinal fibers, as well as, in ALS, in brainstem nuclei of degenerating neurons.

Recently Lloyd et al. [25] reported reductions in regional $\left[{ }^{11} \mathrm{C}\right]$-flumazenil volumes of distribution (an index of neuronal density in pyramidal cells and interneurons) in several frontal, parietal and visual association areas, including the motor and premotor cortices, in patients with ALS. Turner et al. using $\left[{ }^{11} \mathrm{C}\right](R)$-PK11195 in ten ALS patients, found widespread microglial activation (an index of neuroinflammatory reaction) in the motor cortex, pons and thalamus, the uptake being positively correlated with the burden of upper motor neuron clinical signs [26]. Astrocytosis has also been shown to increase in regions of neurodegeneration in patients with ALS, suggesting that astrocytes are the main determinant of disease progression 
Table 3 Numerical results of SPM comparisons between ${ }^{18}$ F-FDG uptake in the controls and spinal patients

\begin{tabular}{|c|c|c|c|c|c|c|c|}
\hline \multirow[t]{2}{*}{ Comparison } & \multicolumn{3}{|l|}{ Cluster level } & \multicolumn{4}{|l|}{ Voxel level } \\
\hline & $\begin{array}{l}\text { Cluster extent } \\
\text { (no. of voxels) }\end{array}$ & $\begin{array}{l}\text { Corrected } \\
p \text { value }^{\mathrm{a}}\end{array}$ & $\begin{array}{l}\text { Cortical region } \\
\text { of voxel }\end{array}$ & Maximum $Z$ score & $\begin{array}{l}\text { Talairach } \\
\text { coordinates }\end{array}$ & $\begin{array}{l}\text { Cortical } \\
\text { region }\end{array}$ & $\mathrm{BA}^{\mathrm{b}}$ \\
\hline \multirow{5}{*}{$\begin{array}{l}\text { Controls vs. } \\
\text { spinal patients }\end{array}$} & \multirow[t]{3}{*}{786} & \multirow[t]{3}{*}{0.000} & Right cerebellum & 4.07 & $2,-70,-8$ & & \\
\hline & & & Left occipital & 3.73 & $-5,-74,-5$ & Lingual gyrus & 18 \\
\hline & & & Left occipital & 3.4 & $-5,-89,-10$ & Lingual gyrus & 18 \\
\hline & \multirow[t]{2}{*}{238} & \multirow[t]{2}{*}{0.02} & Right occipital & 3.83 & $19,-94,-19$ & Fusiform gyrus & 18 \\
\hline & & & Right occipital & 3.65 & $23,-79,-12$ & Fusiform gyrus & 19 \\
\hline \multirow{2}{*}{$\begin{array}{l}\text { Spinal patients } \\
\text { vs. controls }\end{array}$} & \multirow[t]{2}{*}{191} & \multirow[t]{2}{*}{0.05} & Right brainstem & 4.51 & $7,-19,-17$ & Midbrain & \\
\hline & & & Right brainstem & 3.9 & $9,-23,-34$ & Pons & \\
\hline
\end{tabular}

${ }^{\text {a }}$ Threshold $p<0.001$, uncorrected at voxel level. A value of $\leq 0.05$, corrected for multiple comparison at the cluster level, was accepted as statistically significant.

${ }^{\mathrm{b}}$ The corresponding cortical region and BA are reported for each significant cluster. If the maximum correlation is achieved outside the grey matter, the nearest grey matter (within a range of $3 \mathrm{~mm}$ ) is indicated with the corresponding BA

in some forms of ALS [27, 28]. Another mechanism that might account for the increased number of astrocytes in brain regions affected by ALS is their role in replacing the dead neurons filling the space left empty by shrinkage of neurons in the end stage of the disorder [29, 30]. These putative local increases in microglia and astrocytes may in turn result in higher ${ }^{18} \mathrm{~F}$-FDG uptake in ALS patients as compared to controls, even though results reporting a positive correlation between microglia activation and glucose metabolism were not consistent [31, 32].
The mechanism of ${ }^{18}$ F-FDG accumulation in the brain has been investigated in several studies highlighting the role of astrocytes as the preferential site of glucose metabolism during brain activation and accounting for about $50 \%$ of the glucose consumption at rest [33-35]. In this model glutamate-induced glycolysis in astrocytes provides lactate as a substrate for neuronal firing. Activated astrocytes, by taking up glucose directly from intraparenchymal capillaries, couple synaptic activity to energy metabolism. Under glutamate excess, such a neurometa-

Table 4 Numerical results of SPM comparisons between ${ }^{18}$ F-FDG uptake in the controls and bulbar patients

\begin{tabular}{|c|c|c|c|c|c|c|c|}
\hline \multirow[t]{2}{*}{ Comparison } & \multicolumn{3}{|l|}{ Cluster level } & \multicolumn{4}{|l|}{ Voxel level } \\
\hline & $\begin{array}{l}\text { Cluster extent } \\
\text { (no. of voxels) }\end{array}$ & $\begin{array}{l}\text { Corrected } \\
p \text { value }^{\mathrm{a}}\end{array}$ & $\begin{array}{l}\text { Cortical region } \\
\text { of voxel }\end{array}$ & Maximum $Z$ score & $\begin{array}{l}\text { Talairach } \\
\text { coordinates }\end{array}$ & Cortical region ${ }^{\mathrm{b}}$ & $\mathrm{BA}^{\mathrm{b}}$ \\
\hline \multirow{12}{*}{$\begin{array}{l}\text { Controls vs. } \\
\text { bulbar patients }\end{array}$} & \multirow[t]{12}{*}{20,094} & \multirow[t]{12}{*}{0.000} & Left frontal & 5.46 & $-44,4,44$ & Middle frontal gyrus & 6 \\
\hline & & & Left frontal & 4.51 & $-30,33,-2$ & Inferior frontal gyrus & 47 \\
\hline & & & Left frontal & 4.22 & $-40,7,35$ & Precentral gyrus & 9 \\
\hline & & & Left frontal & 4.17 & $-46,25,19$ & Middle frontal gyrus & 46 \\
\hline & & & Right sublobar & 5.16 & $40,23,16$ & Insula & 13 \\
\hline & & & Right frontal & 4.93 & $47,-4,46$ & Precentral gyrus & 6 \\
\hline & & & Right frontal & 4.76 & $51,7,29$ & Inferior frontal gyrus & 9 \\
\hline & & & Right frontal & 4.60 & $53,6,17$ & Inferior frontal gyrus & 44 \\
\hline & & & Right frontal & 4.51 & $29,32,-1$ & Inferior frontal gyrus & 47 \\
\hline & & & Right frontal & 4.41 & $6,33,47$ & Superior frontal gyrus & 8 \\
\hline & & & Right frontal & 4.13 & $45,33,15$ & Middle frontal gyrus & 46 \\
\hline & & & Right limbic & 4.07 & $2,9,32$ & Cingulate gyrus & 24 \\
\hline \multirow{2}{*}{$\begin{array}{l}\text { Bulbar patients } \\
\text { vs. controls }\end{array}$} & 75 & 0.024 & Right brainstem & 4.88 & $7,-16,-23$ & Pons & \\
\hline & 124 & 0.004 & Left brainstem & 4.42 & $-8,-25,-29$ & Pons & \\
\hline
\end{tabular}

${ }^{\text {a }}$ Threshold $p<0.05$ corrected for multiple comparisons with the FDR at the voxel level. A value of $\leq 0.05$, corrected for multiple comparison at the cluster level, was accepted as statistically significant.

${ }^{\mathrm{b}}$ The corresponding cortical region and BA are reported for each significant cluster. If the maximum correlation is achieved outside the grey matter, the nearest grey matter (within a range of $3 \mathrm{~mm}$ ) is indicated with the corresponding BA 


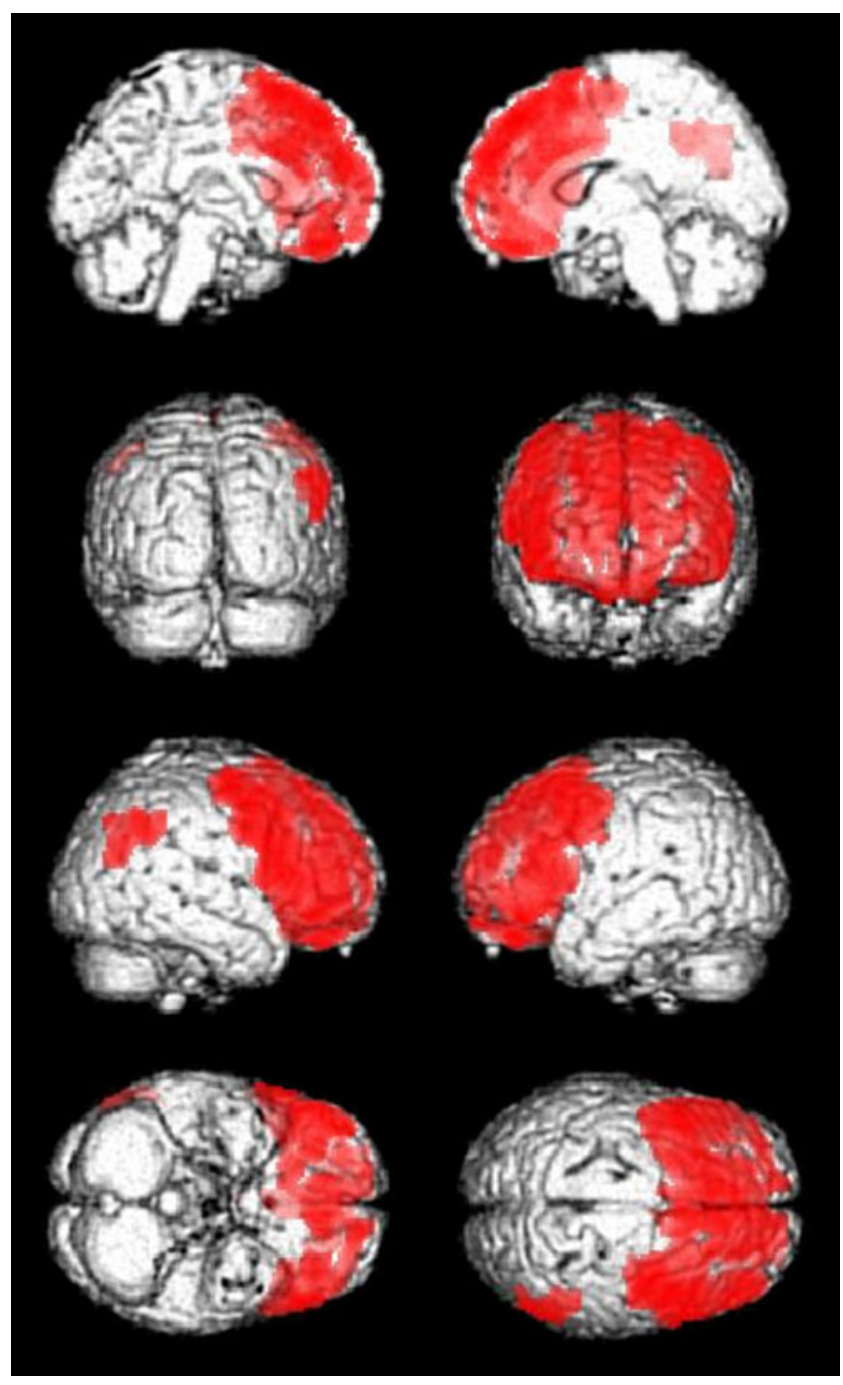

Fig. 3 Three-dimensional rendering showing those regions in which ${ }^{18}$ F-FDG uptake was significantly higher in CTR $(n=22)$ as compared with BUL $(n=13)$ (threshold $p<0.05$, corrected for multiple comparisons with the FDR at voxel level): top row left medial left view; top row right medial right view; second row left posterior view; second row right frontal view; third row left right-side view; third row right left-side view; bottom row left view from below; bottom row right view from above. Coordinate and regional details are presented in Table 4

bolic loop might cause an increase in ${ }^{18} \mathrm{~F}-\mathrm{FDG}$ accumulation in ALS, even in the presence of neuronal degeneration.

Abe et al. [36] suggested that ALS patients can be divided into groups based on their specific cognitive impairment. Impairment of executive functions has been reported in ALS patients, accounting for 5 to $44 \%$ of cases $[12,22]$. Verbal fluency has been regarded as the most effective neuropsychological test in identifying cognitively impaired patients [37]. This finding is confirmed by our results showing a significant difference in executive test scores between patients with bulbar and spinal onset (Table 1). Patients with bulbar onset scored worse than patients with spinal onset in the FAS test, Stroop word test (impaired attention), and TMT-B and B-A tests (indicating poorer sustained and switching attention). Poorer scores in the Wisconsin and other Stroop tests did not reach statistical significance. Such a profile is consistent with moderately impaired frontal lobe function in patients with bulbar onset. Furthermore, the relative decrease in ${ }^{18} \mathrm{~F}$-FDG in the extramotor cortex comparing all ALS patients with control subjects was mostly due to its presence in the patients with bulbar onset in the former group. This was confirmed by the highly significant hypometabolism in the prefrontal and frontal cortex in the patients with bulbar onset as compared to those with spinal onset, in accordance with the results of previous studies showing evidence of diffuse cortical involvement in patients with both upper and lower motorneuron disease [17, 18, 38]. Our findings support the growing evidence of cognitive and extramotor changes in bulbar pathology [39] and seem to be in favour of different neuropsychological and functional patterns in ALS patients between those with bulbar and spinal onset.

The use of a group of subjects with negative PET scans as a control group is suboptimal. However, we underline that the control group was specifically set up for this study and the same protocol and scanner were used in both patients and controls. Furthermore, the stringent exclusion criteria were the same as those applied in previous investigations into other neurodegenerative disorders [40, 41]. On the other hand, investigating neurologically normal subjects undergoing a PET scan for other reasons, and also negative for any disease, avoids the exposure of healthy individuals to radiation and makes the technology available to most PET centres sparing the costs and effort necessary to build up control groups of completely healthy subjects [42].

Another issue is the rather unexpected finding of relative hypermetabolism in neurodegenerative diseases. It has been argued that such hypermetabolism might be an artefact of global mean normalization in SPM, and hence some alternative normalization procedures have been suggested [43]. For instance, cerebellar or thalamic normalization would result in the inclusion of only the highest uptake values in the reference value, but these procedures would require an a priori assumption, namely that neither of the two structures shall be affected by hypo- or hypermetabolism, a condition that does not necessarily apply to ALS. We approached the problem by allowing the normalization value to incorporate only those voxels with a value higher than the global mean (grey matter threshold of 1.0), thus minimizing the risk of artefactual hypermetabolism [24]. The result of our test confirmed the choice made about the default threshold (0.8), since both FDR-corrected significances and Zscores were confirmed in all comparisons performed with a grey matter threshold of 1.0 . 


\section{Conclusion}

The results of the study involving the largest cohort of ALS patients studied by PET provide evidence of both relatively increased and decreased metabolism in various areas of the brain in patients with either bulbar or spinal onset. Relatively increased midbrain metabolism was found for the first time in both groups and appears to be the metabolic trait in ALS, although this finding needs to be confirmed by quantification of absolute glucose consumption. Reduced relative prefrontal and frontal metabolism is exclusive to patients with bulbar onset who were suffering from significantly reduced verbal fluency, confirming the higher rate of frontal impairment in this group.

Acknowledgments The authors wish to thank the patients who kindly agreed to participate in this study, Ms Deborah Bertoluzzo and the personnel of IRMET for their valuable help in patient management, and Mrs Emanuela Enrico for English editing. This work was in part supported by Compagnia di San Paolo, Programma Neuroscienze 2008-2009 (to Consuelo Valentini e Andrea Calvo), and by the European Community's Health Seventh Framework Programme (FP7/2007-2013) to Adriano Chiò (grant 259867).

Conflicts of interest None.

\section{References}

1. Brooks BR, Miller RG, Swash M, Munsat TL. El Escorial revisited: revised criteria for the diagnosis of amyotrophic lateral sclerosis. Amyotroph Lateral Scler Other Motor Neuron Disord. 2000;1:293-9.

2. Chiò A, Mora G, Calvo A, Mazzini L, Bottacchi E, Mutani R, et al. Epidemiology of ALS in Italy: a 10-year prospective population-based study. Neurology. 2009;72:725-31.

3. Leigh PN, Kew JJM, Goldstein LH, Brooks DJ. The cerebral lesions in amyotrophic lateral sclerosis: new insights from pathology and functional brain imaging. In: Rose C, editor. Amyotrophic lateral sclerosis from Charcot to the present and into the future. London: Smith-Gordon; 1994. p. 191-209.

4. Talbot PR, Goulding PJ, Lloyd JJ, Snowden JS, Neary D, Testa HJ. Inter-relation between 'classic' motor neuron disease and frontotemporal dementia: neuropsychological and single photon emission computed tomography study. J Neurol Neurosurg Psychiatry. 1995;58:541-7.

5. Geser F, Brandmeir NJ, Kwong LK, Martinez-Lage M, Elman L, McCluskey L, et al. Evidence of multisystem disorder in wholebrain map of pathological TDP-43 in amyotrophic lateral sclerosis. Arch Neurol. 2008;65:636-41.

6. Gallassi R, Montagna P, Morreale A, Lorusso S, Tinuper P, Daidone R, et al. Neuropsychological, electroencephalogram and brain computed tomography findings in motor neuron disease. Eur Neurol. 1989;29:115-20.

7. Ludolph AC, Langen KJ, Regard M, Herzog H, Kemper B, Kuwert $\mathrm{T}$, et al. Frontal lobe function in amyotrophic lateral sclerosis: a neuropsychologic and positron emission tomography study. Acta Neurol Scand. 1992;85:81-9.

8. Iwasaki Y, Kinoshita M, Oceda K, Takamiya K, Shiojima T. Cognitive impairment in amyotrophic lateral sclerosis and its relation to motor disabilities. Acta Neurol Scand. 1990;81:141-3.
9. Kew JJM, Goldstein LH, Leigh PN, Abrahams S, Cosgrave N, Passingham RE, et al. The relationship between abnormalities of cognitive function and cerebral activation in amyotrophic lateral sclerosis. A neuropsychological and positron emission tomography study. Brain. 1993;116:1399-423.

10. Abrahams S, Leigh PN, Kew JJ, Goldstein LH, Lloyd CM, Brooks DJ. A positron emission tomography study of frontal lobe function (verbal fluency) in amyotrophic lateral sclerosis. J Neurol Sci. 1995;129(Suppl):44-6.

11. Abe K, Fujimura H, Toyooka K, Sakoda S, Yorifuji S, Yanagihara T. Cognitive function in amyotrophic lateral sclerosis. J Neurol Sci. 1997;148:95-100.

12. Abrahams S, Goldstein LH, Suckling J, Ng V, Simmons A, Chitnis X, et al. Frontotemporal white matter changes in amyotrophic lateral sclerosis. J Neurol. 2005;252:321-31.

13. Andreadou E, Sgouropoulos P, Varelas P, Gouliamos A, Papageorgiou C. Subcortical frontal lesions on MRI in patients with motor neurone disease. Neuroradiology. 1998;40:298-302.

14. Kato S, Hayashi H, Yagishita A. Involvement of the frontotemporal lobe and limbic system in amyotrophic lateral sclerosis: as assessed by serial computed tomography and magnetic resonance imaging. J Neurol Sci. 1993;116:52-8.

15. Peavy GM, Herzog AG, Rubin NP, Mesulam M-M. Neuropsychological aspects of dementia of motor neuron disease: a report of two cases. Neurology. 1992;42:1004-8.

16. Hudson AJ. Amyotrophic lateral sclerosis and its association with dementia, parkinsonism and other neurological disorders: a review. Brain. 1981;104:217-47.

17. Dalakas MC, Hatazawa J, Brooks RA, Di Chiro G. Lowered cerebral glucose utilization in amyotrophic lateral sclerosis. Ann Neurol. 1987;22:580-6.

18. Hatazawa J, Brooks RA, Dalakas MC, Mansi L, Di Ghiro G. Cortical moto-sensory hypometabolism in amyotrophic lateral sclerosis: a PET study. J Comput Assist Tomogr. 1988;12:630-6.

19. Le Forestier N, Maisonobe T, Spelle L, Lesort A, Salachas F, Lacomblez L, et al. Primary lateral sclerosis: further clarification. J Neurol Sci. 2001;185:95-100.

20. Turner MR, Hammers A, Al-Chalabi A, Shaw CE, Andersen PM, Brooks DJ, et al. Cortical involvement in four cases of primary lateral sclerosis using [(11)C]-flumazenil PET. J Neurol. 2007;254:1033-6.

21. Claassen DO, Josephs KA, Peller PJ. The stripe of primary lateral sclerosis: focal primary motor cortex hypometabolism seen on fluorodeoxyglucose F18 positron emission tomography. Arch Neurol. 2010;67:122-5.

22. Strong MJ, Grace GM, Freedman M, Lomen-Hoerth C, Woolley $\mathrm{S}$, Goldstein LH, et al. Consensus criteria for the diagnosis of frontotemporal cognitive and behavioral syndromes in amyotrophic lateral sclerosis. Amyotroph Lateral Scler Other Motor Neuron Disord. 2009;10:131-46.

23. Bennett CM, Wolford GL, Miller MB. The principled control of false positives in neuroimaging. Soc Cogn Affect Neurosci. 2009;4:417-22.

24. Yatham LN, Liddle PF, Lam RW, Zis AP, Stoessl AJ, Sossi V, et al. Effect of electroconvulsive therapy on brain 5-HT2 receptors in major depression. Br J Psychiatry. 2010;196:474-9.

25. Lloyd CM, Richardson MP, Brooks DJ, Al-Chalabi A, Leigh PN. Extramotor involvement in ALS: PET studies with the GABA(A) ligand [(11)C]flumazenil. Brain. 2000;123:2289-96.

26. Turner BJ, Lopes EC, Cheema SS. The serotonin precursor 5hydroxytryptophan delays neuromuscular disease in murine familial amyotrophic lateral sclerosis. Amyotroph Lateral Scler Other Motor Neuron Disord. 2003;4:171-6.

27. Aquilonius SM, Jossan SS, Ekblom JG, Askmark H, Gillberg PG. Increased binding of 3H-L-deprenyl in spinal cords from patients with amyotrophic lateral sclerosis as demonstrated by autoradiography. J Neural Transm Gen Sect. 1992;89:111-22. 
28. Yamanaka K, Chun SJ, Boillee S, Fujimori-Tonou N, Yamashita H, Gutmann DH, et al. Astrocytes as determinants of disease progression in inherited amyotrophic lateral sclerosis. Nat Neurosci. 2008;11:251-3.

29. Yu I, Inaji M, Maeda J, Okauchi T, Nariai T, Ohno T, et al. Glial cell-mediated deterioration and repair of the nervous system after traumatic brain injury in a rat model as assessed by positron emission tomography. J Neurotrauma. 2010;27:1463-75.

30. Marik J, Ogasawara A, Martin-McNulty B, Ross J, Flores JE, Gill HS, et al. PET of glial metabolism using 2-18F-fluoroacetate. J Nucl Med. 2009;50:982-90

31. Yokokura M, Mori N, Yagi S, Yoshikawa E, Kikuchi M, Yoshihara Y, et al. In vivo changes in microglial activation and amyloid deposits in brain regions with hypometabolism in Alzheimer's disease. Eur J Nucl Med Mol Imaging. 2011;38:343-51.

32. Fukumoto D, Hosoya T, Nishiyama S, Harada N, Iwata $H$, Yamamoto S, et al. Multiparametric assessment of acute and subacute ischemic neuronal damage: a small animal positron emission tomography study with rat photochemically induced thrombosis model. Synapse. 2011;65:207-14.

33. Barros LF, Porras OH, Bittner CX. Why glucose transport in the brain matters for PET. Trends Neurosci. 2005;28:117-9.

34. Magistretti PJ. Cellular bases of functional brain imaging: insights from neuron-glia metabolic coupling. Brain Res. 2000;886:108-12.

35. Nehlig A, Coles J. Cellular pathways of energy metabolism in the brain: is glucose used by neurons or astrocytes? Glia. 2007;55:1238-50.
36. Abe K, Fujimura H, Toyooka K, Hazama T, Hirono N, Yorifuji S, et al. Single-photon emission computed tomographic investigation of patients with motor neuron disease. Neurology. 1993;43:1569-73.

37. Abrahams S, Leigh PN, Harvey A, Vythelingum GN, Grise D, Goldstein LH. Verbal fluency and executive dysfunction in amyotrophic lateral sclerosis (ALS). Neuropsychologia. 2000;38:734-47.

38. Abrahams S, Goldstein LH, Kew JJM, Brooks DJ, Lloyd CM, Frith $\mathrm{CD}$, et al. Frontal lobe dysfunction in amyotrophic lateral sclerosis. A PET study. Brain. 1996;119:2105-20.

39. Abrahams S, Goldstein LH, Al-Chalabi A, Pickering A, Morris RG, Passingham RE, et al. Relation between cognitive dysfunction and pseudobulbar palsy in amyotrophic lateral sclerosis. J Neurol Neurosurg Psychiatry. 1997;62:464-72.

40. Pagani M, Dessi B, Morbelli S, Brugnolo A, Salmaso D, Piccini A, et al. MCI patients declining and not-declining at mid-term follow-up: FDG-PET findings. Curr Alzheimer Res. 2010;7:287-94.

41. Nobili F, Mazzei D, Dessi B, Morbelli S, Brugnolo A, Barbieri P, et al. Unawareness of memory deficit in amnestic MCI: FDG-PET findings. J Alzheimers Dis. 2010;22:993-1003.

42. Del Sole A, Clerici F, Chiti A, Lecchi M, Mariani C, Maggiore L, et al. Individual cerebral metabolic deficits in Alzheimer's disease and amnestic mild cognitive impairment: an FDG PET study. Eur J Nucl Med Mol Imaging. 2008;35:1357-66.

43. Borghammer P, Cumming P, Aanerud J, Gjedde A. Artefactual subcortical hyperperfusion in PET studies normalized to global mean: lessons from Parkinson's disease. Neuroimage. 2009;45:249-57. 